\title{
Numerical modeling of radiative heat and mass transfer for sweet potato during drying
}

\begin{abstract}
In this study, a numerical model was developed to accurately describe the changes in moisture content and temperature distribution of sweet potato during infrared drying, with the consideration of shrinkage-dependent diffusivity and evaporation phenomena. The couple heat and mass transfer and 2D axisymmetric simulations were done using COMSOL Multiphysics. The simulation results were further evaluated based on experimental data. Sensitivity analysis was also conducted and the effects of different simulation parameters on the drying process were presented. The results showed that the developed model considering shrinkage diffusivity and evaporation adequately described the drying process of sweet potato undergoing infrared drying. The mass transfer coefficient, heat transfer coefficient, shrinkage-dependent diffusivity, and infrared heat energy greatly influenced the moisture distribution during the drying process of sweet potato. The temperature distribution during the infrared drying of sweet potato was highly sensitive to the infrared heating energy.
\end{abstract}

Keyword: Sweet potato; COMSOL Multiphysics 